\title{
V.S. Tomelleri, Linguistica e filologia in Unione Sovietica. Trilogia fra sapere epotere, Mimesis, Milano-Udine 2020 (= Eterotopie, 667), pp. 170.
}

Prima ancora che per la scorrevolezza dello stile, il nitore dell'esposizione, l'originalità del contenuto e la significatività dei risultati ottenuti, la recente monografia di Vittorio Springfield Tomelleri è un lavoro prezioso e degno della massima attenzione critica perché sostanzialmente unico nel campo della slavistica italiana: un viaggio saggistico attraversato dalla capacità non comune di individuare, in maniera tangenziale e complementare, un network di interazioni fra storie ed eventi solo apparentemente atomizzati e isolati in sé stessi ma, in realtà, figli di un medesimo orizzonte socioculturale. Originariamente concepite come relazioni autonome per, rispettivamente, la viII Giornata di studi armeni e caucasici, il vi Congresso italiano di Slavistica e il XVI Congresso internazionale degli Slavisti (intervento, quest'ultimo, successivamente ritirato), le sezioni che compongono l'autoproclamata trilogia, oggi edita in uno snello volume per i tipi di Mimesis, costituiscono infatti - più che elementi di una triade hegeliana - tappe cronologicamente consequenziali di un più ampio discorso unitario sul rapporto storico e ideologico fra potere e ricerca umanistica, uno dei filoni di studio su cui l'attenzione scientifica dell'autore si è maggiormente soffermata nel corso dell'ultimo decennio. Si tratta di un rapporto che al tempo della società liquida si ha gioco facile a definire conflittuale (è sotto gli occhi di tutti il discredito mediatico al quale la comunità scientifica delle democrazie liberali occidentali viene sistematicamente sottoposta), ma che assume contorni ancora più frastagliati nello spazio apertamente ideologico del totalitarismo staliniano, dove l'esigenza di costruire un'identità autoctona in antagonismo più o meno manifesto a quella occidentale dovette fare i conti con la costante e coatta limitazione delle libertà individuali - ivi compresa la cooperazione scientifica internazionale - imposta dalla ragion di Stato.

Il primo capitolo, intitolato Ex Oriente lux? Linguistica slava fra Europa orientale e occidentale, è un ambizioso excursus storico sull'evoluzione parallela di due paradigmi scientifici in rapporto di alterità dialettica, la linguistica slava e quella occidentale, alla vigilia di due rivoluzioni distinte ma complementari: da una parte quella metodologica dello strutturalismo saussuriano, dall'altro quella politica dell'Ottobre bolscevico. L'apparizione del Cours de linguistique générale (o, più propriamente, del testo approntato a posteriori da Charles Bally e Albert Sechehaye) è sintesi e primo punto di arrivo di un secolo di profonde trasformazioni euristiche in grado di cambiare volto alla linguistica occidentale: dall'emancipazione del metodo storico-comparativo dei neogrammatici rispetto alla filologia classica al successivo spostamento dell'interesse scientifico verso le lingue vive, la dimensione del parlato e la microvariazione dialettale. A tutto questo la linguistica slava, va detto, 
non rimane affatto estranea: esemplare, per quanto ancora misconosciuta, è l'innegabile influenza esercitata sul pensiero saussuriano dalla scuola di Jan Baudouin de Courtenay, la cui pluridecennale attività di ricerca, per avverse contingenze linguistiche e geografiche (russo e polacco non avevano allora né il prestigio né tantomeno la diffusione del francese), non poté tuttavia godere di una cassa di risonanza paragonabile a quella di cui beneficiò il linguista ginevrino. Ma l'eredità negletta di Baudouin de Courtenay, peraltro nemmeno citato nel Cours, è solo l'ultimo anello di una lunga catena che sembrerebbe quasi tracciare un invalicabile solco comunicativo, per non dire epistemologico, tra linguistica slava e occidentale, a dispetto delle non poche affinità nel percorso evolutivo di entrambe le discipline tra XIX e primi decenni del XX secolo: si pensi agli studi sul linguaggio poetico dell'Opojaz pietrogradese, vera incubatrice del formalismo, o ancora al fiorire delle indagini etno-dialettologiche promosse dal Circolo linguistico moscovita, che ispireranno concetti - come quelli, trubeckojani, di contatto linguistico e jazykovoj sojuz - destinati a mettere in discussione l'egemonia dello Stammbaum schleicheriano e a divenire centrali nella tipologia linguistica dei decenni successivi. È precisamente nel delineare le connessioni storiche fra le ricerche di linguistica areale di Baudouin de Courtenay e Trubeckoj che Tomelleri introduce per la prima volta nel testo il nome di Nikolaj Jakovlevič Marr: un accostamento che, per ammissione dello stesso autore, "[...] può parere avventato, per non dire offensivo" (p. 27), ma che dev'essere in realtà inquadrato nell'ottica di quel vuoto di potere simbolico che l' irreversibile crisi post-bellica dell' individualismo positivista spalanca in favore di istanze sociali contrassegnate da un più pronunciato egalitarismo collettivistico. Così come il circolo praghese di Jakobson e Trubeckoj operava come un sol corpo scientifico, senza lasciare margine di manovra per individualismi e correnti deviazionistiche, così Marr, convertito sulla via di Damasco ad una fantalinguistica 'prometeica' apertamente anticolonialista (in senso antioccidentale), si trovò ad essere inconsapevole megafono dell' internazionalismo marxista-leninista, nume tutelare nella precoce saldatura di regime fra scienza e ideologia e nella transizione delle discipline linguistiche sovietiche da scienze umanistiche (gumanitarnye) a sociali (ob̌̌čstvennye). È nello spazio artificiosamente ideologico di una 'nuova' linguistica sovietica come strumento del proletariato contro la precedente tradizione filologica e la linguistica borghese occidentale che va interpretata la rinnovata attenzione di Marr verso lo studio delle lingue vive, dei dialetti privi di scrittura, del contatto linguistico e della variazione diastratica applicata al concetto di classe (anche se, come puntualmente rileva Tomelleri in più passaggi, lo stesso Marr aveva una concezione di 'classe' piuttosto eterodossa): l'utopico fine ultimo sarebbe stato la creazione di una lingua universale in grado di servire la società del futuro, aclassista e socialista.

Sulla figura di Marr e la sua peculiare attività, quasi impossibili da afferrare in tutta la loro polimorfica complessità, è incentrato il secondo capitolo, intitolato N.Ja. Marr filologo e linguista militante. Un enigma solubile?. Si tratta di un saggio interessante anche per il tono, rispettosamente imparziale ed equilibrato, che Tomelleri sceglie di adottare nell'esposizione di meriti e demeriti scientifici dello studioso georgiano. Su Marr in letteratura si è detto tutto e il contrario di tutto: ambizioso burocrate, strampalato opportunista, folle ciarlatano o, conversamente, visionario nietzschiano dotato di una magnetica personalità e di una disciplina di ferro, sociolinguista ante litteram, addirittura filosofo del linguaggio sui generis (p. 49). Enigmatiche e non lineari sono in effetti le tappe di genesi, ascesa e rovinosa caduta postuma del mito marriano all'ombra del totalitarismo staliniano. Assunto agli onori della comunità scientifica internazionale, agli albori del xx secolo, per i fortunati scavi archeologici ad Ani, capitale medievale del regno armeno, e per aver scoperto e successivamente pubblicato la traduzione georgiana del primo commento al Cantico dei Cantici di Ippolito di Roma (p. 70), il nome di Marr è tuttavia legato a doppio filo alla sua pseu- 
doteoria glottogonica (jafetičeskaja teorija), i cui lineamenti, popolarizzati nella cosiddetta Nuova dottrina del linguaggio (Novoe učenie ob jazyke) e in una sterminata serie di articoli e pubblicazioni monografiche, vennero costantemente rimaneggiati nel corso di quattro decenni. Non è semplice stabilire come e perché, ad un certo punto, Marr decise di investire tutte le proprie energie scientifiche ed organizzative a supporto dell' impresa donchisciottesca della teoria jafetica che, muovendo da un'indimostrabile origine comune delle lingue cartveliche e semitiche, arrivava a postulare un'associazione storicamente consistente fra strati linguistici e conflitto interclassista. Come nota lo stesso Tomelleri (pp. 33, 79-80), oltre al nuovo clima culturale postrivoluzionario e all'ottemperanza al principio staliniano della presenza di un'autorità centrale in ogni scienza in grado di formare stuoli di discepoli acritici (azzeccato, qui, il paragone fra il marrismo e il lysenkoismo), ebbero un peso determinante l'anelito marriano alla creazione di una nuova scienza genuinamente sovietica e, forse, le conseguenze psicologiche derivanti da una serie di pesanti rovesci personali e professionali: in primis la perdita di tutto il materiale legato agli scavi di Ani e il completo rigetto della teoria jafetica nella linguistica occidentale, ma anche l'avvertita necessità di una riabilitazione storica ed etnica delle lingue cartveliche nei confronti degli 'usurpatori' indoeuropei e la morte prematura del figlio Vladimir (forse il durissimo giudizio di Trubeckoj sulla 'sanità mentale' di Marr, di cui si legge a p. 27 , non cadeva poi così lontano dal bersaglio). Ugualmente non limpida è la cifra delle motivazioni politiche che spinsero Stalin in persona, nell'estate del 1950, a sconfessare i presupposti teorici del marrismo, dopo decenni di predominio ideologico e liquidazione (anche fisica) degli oppositori: determinante fu forse l'ondata di risorgente nazionalismo scaturito dal mito del Den' pobedy, in aperta contrapposizione al cosmopolismo internazionalista di Marr, o la necessità di coalizzare un fronte panslavo a guida sovietica in funzione antioccidentale, dimenticando per strada la lotta di classe così centrale nella teoria jafetica (pp. 59-60). Il sottoscritto aggiungerebbe - ipotesi su cui peraltro anche l'autore concorda - l'esigenza di stringere buone relazioni diplomatiche con la neonata Repubblica Popolare Cinese di Máo Zédōng: dettami di Realpolitik che si scontravano con le elucubrazioni dello schema glottogonico jafetico, nel quale il cinese era classificato al primo stadio, quello meno evoluto e più vicino al supposto protolinguaggio originario, magico-sillabico. Quali che fossero le reali intenzioni di Stalin, in ogni caso, la precoce damnatio memoriae subita da Marr denuncia la congenita fragilità del suo paradigma epistemologico, incapace di tenere il passo di una pars destruens veemente e a tratti paranoica, ma non priva di punti di forza (si pensi ai giustificati strali contro il razzismo strisciante di certa indoeuropeistica 'romantica' o alla concezione della lingua come sovrastruttura generata dall'interazione dialettica sociale, idea ripresa - sebbene con rigore scientifico del tutto diverso - da studiosi del calibro di Ferruccio Rossi-Landi).

Apparentemente provocatorio, ma in realtà esposto con grande coerenza argomentativa, è infine il terzo capitolo, intitolato N.Ja. Marr e D.S. Lichačëv: parallelismi che si incontrano?. Il paragone, come mette da subito in chiaro Tomelleri (p. I03), non si dà tanto fra i due studiosi - pure accomunati da interessanti contatti biografici nell'evoluzione dei rispettivi rapporti col potere costituito quanto fra le discipline cui sono associati (il Novoe učenie ob jazyke e la Tekstologija), specialmente nell'opposizione dialettica di cui si fanno portatrici verso la linguistica storico-comparativa da un lato, verso la critica del testo occidentale dall'altro. Se Marr aveva rifiutato i meccanicismi formali della scuola neogrammatica, concentrandosi sull'apporto semantico e latamente ideologico delle unità linguistiche, Lichačev opponeva al supposto antistoricismo della critica del testo occidentale un approccio dialettico alle peculiarità della letteratura antico russa, che intendesse le trasformazioni di un testo come "prodotto storico dell'attività umana" (p. IIo), ovvero come "redazione" (redakcija) risultante dalla somma di interventi esterni collettivi (nel senso antiindividualista del termine), con- 
sapevoli, psicologicamente motivati, qualitativamente immersi nel (e figli del) proprio spaziotempo. È questa un'impostazione con la quale Tomelleri, per la prima volta nel volume, dialoga criticamente in prima persona, sottolineando i punti deboli di un impianto ideologico che sembra ignorare la questione della gradazione qualitativa degli errori nelle copie manoscritte, oltre a sovrastimare la centralità ecdotica di un contesto più ampio ai fini della corretta interpretazione di testi spesso inseriti in codici miscellanei (pp. II6-II7). Questo confronto a distanza sembra rievocare le note polemiche di metodo e merito che, in campo filologico e letterario, contrapposero in diversi anni Lichačev ad esponenti di spicco della slavistica italiana, quali Riccardo Picchio (I923-201I) e Angiolo Danti (1939-1979). Oggi come ieri, ad esempio, è centrale il problema della visione nazionale della letteratura antico russa promosso dalla testologia: come mette giustamente in rilievo Tomelleri (pp. I2I-I22), accanto alla tradizione autoctona di opere originali la gran parte del materiale è costituito da traduzioni di provenienza slavo meridionale (perevodnaja pis'mennost'), il cui carattere di trasmissione tendenzialmente conservativo consiglierebbe l'applicazione non tanto del modello testologico lichačeviano quanto, piuttosto, di quello lachmanniano, più formale e meccanico. In linea con questo apparente paradosso l'opinione generale dell'autore, espressa più volte nel corso del capitolo, è che l'antagonismo epistemologico ricercato e propugnato dalla testologia lichačeviana nei confronti della critica del testo occidentale possa invece, e forse debba, convivere con l'oggetto della sua vis polemica, così come gli aspetti latamente sociolinguistici della teoria jafetica sono complementari al metodo storico-comparativo classico. Un chiaro esempio di questa possibile complementarietà tra visioni, peraltro, è dato dall' intuizione lichačeviana, forse influenzata dal materialismo storico, che le trasformazioni (linguistiche e/o testuali) non avvengano linearmente, ma "a salti" (skačkoobrazno, pp. 125-126): curiosamente ma non troppo, una formulazione simile viene adottata da quelle correnti biolinguistiche contemporanee che postulano il verificarsi di una cesura brusca e traumatica nel processo di evoluzione della facoltà linguistica umana, un 'grande salto in avanti' che avrebbe generato come risultato Merge, l’operazione alla base del programma minimalista.

Menzionare alcuni dei lavori più recenti di Noam Chomsky, Bernd Heine e Tania Kuteva in calce ad un discorso sulla tradizione manoscritta antico russa potrebbe forse sembrare un azzardo privo di senso, ma è semplicemente una delle tante riflessioni collaterali, delle innumerevoli finestre associative che la lettura della monografia di Vittorio Tomelleri, permeata di erudita competenza e dotata di un imponente apparato bibliografico, è in grado di aprire nella mente del lettore. L'augurio, sincero, è che da questo volume possano presto sbocciare ulteriori e ancor più intriganti ramificazioni. 